\title{
Pilot randomised controlled trial of face-down posturing following phacovitrectomy for macular hole
}

D Yorston', MAR Siddiqui', MA Awan', S Walker², ${\text { C } \text { Bunce }^{3} \text { and JW Bainbridge }}^{4}$

\author{
This article has been corrected since Advance Online Publication and a Corrigendum is \\ also printed in this issue
}

Introduction

Macular hole surgery is one of the most successful vitreoretinal interventions. ${ }^{1,2}$ Since the first description by Kelly and Wendel ${ }^{3}$ anatomical success rates have improved, and are now close to $100 \%$. $^{4,5}$ The initial descriptions of macular hole surgery required the patient to assume a face-down posture continuously for a minimum of 1 week. However, this is uncomfortable and inconvenient, and can be associated with complications such as ulnar nerve compression ${ }^{6}$ and angle closure glaucoma. ${ }^{7}$ Some patients may refuse surgery because of concerns about postoperative posturing, and others are unable to maintain a face-down posture for more than brief periods. ${ }^{8}$ Within a few years of the original description of macular hole surgery, other authors were describing successful surgery without postoperative posturing. ${ }^{9}$ Overall, there has been a tendency for the duration of posturing to be shortened, particularly when vitrectomy is combined with phacoemulsification and intraocular lens insertion, ${ }^{5,10-15}$ but there is no definitive proof that posturing for any duration is beneficial. A randomised controlled trial of face down $v$ s seated posture postoperatively showed that there was benefit from face-down posturing, but this benefit was confined to holes with a diameter greater than $400 \mu \mathrm{m} .{ }^{16}$ Although this was the largest trial of face-down posturing to date, the question remains open, because the trial used a variety of different gases for intraocular tamponade, and because some patients had simultaneous cataract surgery, whereas others had vitrectomy alone. Another recently published trial examined the
${ }^{1}$ Vitreo-retinal service, Tennent Institute of Ophthalmology, Gartnavel General Hospital,

Glasgow, UK

${ }^{2}$ Advanced Healthcare Scientist, Electro Diagnostic Imaging Unit, Gartnavel General Hospital, Glasgow, UK

${ }^{3}$ Medical Statistician, Moorfields Eye Hospital, London, UK

${ }^{4}$ FRCOphth, Moorfields Eye Hospital, London, UK

Correspondence: D Yorston, Vitreo-retinal service, Tennent Institute of Ophthalmology, Gartnavel General Hospital, 1053 Great Western Road, Glasgow G12 OYN, UK Tel: + 44 (0) 1412111041 Fax: + 44 (0) 1412112054 E-mail: dbyorston@ btinternet.com

Received: 8 March 2011 Accepted in revised form: 18 July 2011

Published online: 23 September 2011

Keywords: macular hole; vitrectomy; posturing phacoemulsification 
effect of posturing in small holes ( $400 \mu \mathrm{m}$ or less), and found no benefit from face-down posturing. ${ }^{17}$ Two recent systematic reviews have emphasised the need for additional randomised controlled trials. $^{8,18}$

Trials of macular hole surgery are complicated by the variety of techniques used. As well as postoperative posturing, other variations include internal limiting membrane (ILM) peeling, the use of dyes to stain the ILM, the type of gas used for intraocular tamponade, and whether cataract surgery is performed simultaneously or sequentially. There is now good evidence that ILM peeling improves anatomical outcomes, ${ }^{19}$ but there is considerable uncertainty over whether or not combining phacoemulsification with vitrectomy may affect the final success rate, particularly in non-posturing surgery. ${ }^{13,20}$ Because of the possible confounding effect of simultaneous cataract surgery,

we carried out two pilot studies. In one study (reported here), all patients had combined cataract surgery and vitrectomy. In the other, patients had vitrectomy followed by cataract surgery. ${ }^{21}$ The purpose of these pilot studies was to estimate the number of patients required to identify a difference in outcome in a definitive trial.

\section{Patients and Methods}

Patients were eligible for recruitment if they were having surgery for a full-thickness stage II-IV macular hole, and were willing and able to posture face down for 10 days after surgery. Exclusion criteria included inability to posture, refusal to be randomised, or the presence of retinal breaks requiring postoperative posturing to prevent retinal detachment. All patients had preoperative optical coherence tomography (OCT), with measurement of the hole diameter using a Heidelberg Spectralis (Heidelberg Engineering, Heidelberg, Germany). The primary outcome was anatomical closure of the macular hole, defined as an attached macula with re-approximation of the edges of the hole, assessed from postoperative OCT by a specialist vitreoretinal surgeon (JB) who was masked to the treatment allocation. Holes were graded as 'closed', 'flat open', and 'open elevated'. Secondary outcomes included corrected visual acuity at 6 months after surgery, and postoperative complications. All patients had combined surgery. Randomisation was carried out in the operating theatre at the conclusion of surgery, using sealed envelopes. Surgeons were not masked to treatment allocation as they were responsible for instructing the patient about postoperative care. No attempt was made to monitor patient compliance with posturing instructions. All patients were treated according to randomised treatment allocation.

\section{Surgical technique}

A routine phacoemulsification with intraocular lens insertion was carried out. A core vitrectomy was performed, with 20G instruments. The posterior hyaloid face was elevated from the retina in stage II and III holes using the vitreous cutter. The ILM was stained with Trypan blue (Membrane Blue, DORC, Zuidland, The Netherlands) under air, and the ILM was peeled completely from around the hole with a radius of at least one disc diameter. A meticulous internal search was completed, and any retinal breaks or tears were treated with cryotherapy. A total air-fluid exchange was carried out, and the eye was filled with $14 \% \mathrm{C}_{3} \mathrm{~F}_{8}$. If there were no retinal breaks that required postoperative posturing, the patient was randomised. Patients with retinal breaks in the temporal, inferior, or nasal quadrants were ineligible for randomisation and were advised to posture for 1 week to provide the maximum support to the retinal break.

Subjects in the posturing group were asked to maintain a face-down position for $50 \mathrm{~min}$ in every hour for 10 days after surgery. Subjects in the no posture group were asked to avoid a face-up position.

Final refraction and visual acuity measurement took place 6 months after surgery.

The trial was approved by the West Glasgow research ethics committee, and was registered with ClinicalTrials.gov, identifier NCT01020760.

All patients were treated in accordance with the tenets of the Declaration of Helsinki.

\section{Results}

Thirty patients were recruited. No patients were lost to follow-up. Preoperative characteristics are shown in Table 1. Baseline preoperative characteristics were similar in the two groups.

Table 1 Preoperative characteristics

\begin{tabular}{lccc}
\hline & All & Face down & No posture \\
\hline Eyes, $n(\%)$ & 30 & $16(53.3)$ & $14(46.7)$ \\
Age, mean (SD), years & $69.6(5.7)$ & $71.1(5.9)$ & $68.0(5.6)$ \\
Age $>75$ years, $n(\%)$ & $6(20)$ & $4(25)$ & $2(14.3)$ \\
Female, $n$ & 29 & 15 & 14 \\
Pre-op VA LogMAR, mean (SD) & $0.94(0.27)$ & $1.02(0.29)$ & $0.86(0.24)$ \\
VA $>6 / 36, n(\%)$ & $7(23.3)$ & $3(18.8)$ & $4(28.6)$ \\
Duration in months, mean (SD) & $7.5(2.8)$ & $7.6(2.4)$ & $7.3(3.3)$ \\
Stage II/III, $n(\%)$ & $27(90)$ & $13(81.2)$ & $14(100)$ \\
Stage IV, $n(\%)$ & $3(10)$ & $3(18.8)$ & 0 \\
Hole diameter, mean (SD) & $390(133)$ & $433(126)$ & $337(126)$ \\
Hole $>400 \mu$ m, $n$ (\%) & $11(36.7)$ & $6(37.5)$ & $5(35.7)$ \\
\hline
\end{tabular}

Abbreviations: pre-op, preoperative; VA, visual acuity. 


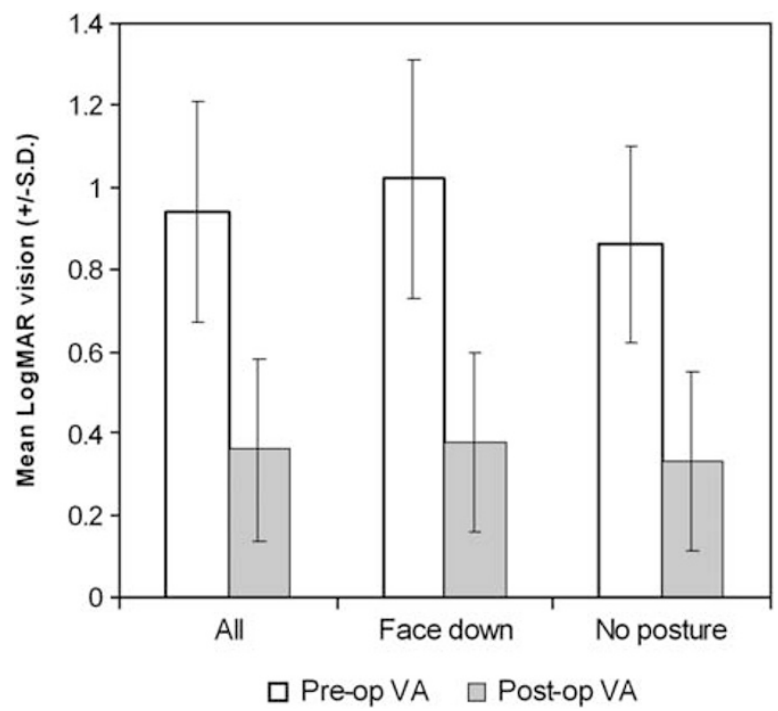

Figure 1 Mean preoperative (pre-op) and postoperative (post-op) visual acuities (VAs).

Postoperatively no patient developed a retinal detachment. There were no complications associated with posturing. One patient in the face-down group developed a pigment epithelial detachment at the fovea, and another in the no posture group lost vision from cystoid macular oedema 6 months after surgery.

The macula hole was closed in all of the no-posture group, and in $15 / 16(93.75 \%$, difference $=6.25 \%$, $95 \% \mathrm{CI}=-16.6$ to $28.9 \%$ ) of the face-down group. The single anatomical failure occurred in an 81-year-old patient who had been symptomatic for about 1 year. Her preoperative vision was $1 / 60$ and the stage III hole had a diameter of $670 \mu \mathrm{m}$.

\section{Visual acuity}

The mean $\log$ MAR vision improved from a mean of 0.94 . (95\% CI $=0.84-1.04)$ preoperatively to 0.36 (95\% $\mathrm{CI}=0.28-0.44$ ) postoperatively (see Figure 1 ). Final visions were similar in the two groups - in the facedown group, 5/16 had a final vision worse than 6/18, compared with $1 / 14$ of the no posture group. The size of the macular hole made little difference to the final visual outcome (Figure 2).

Visual acuity change was slightly greater in the face-down group (0.63 gain) compared with the no posture group ( 0.52 gain, difference $=0.11,95 \%$ $\mathrm{CI}=-0.06$ to 0.28 ). No patient had a final vision less than the preoperative vision.

All eyes in which the hole closed achieved a reading vision of $\mathrm{N} 10$ or better.

Refractive outcomes were acceptable. The mean difference from intended spherical equivalent was -0.16 in the face-down group and -0.18 in the no posture

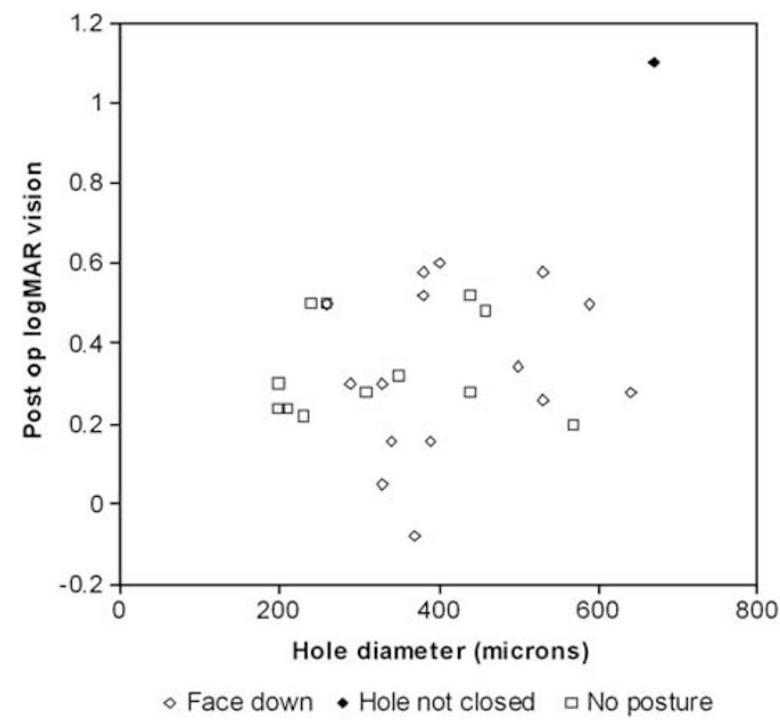

Figure 2 Postoperative vision and hole dinner.

group. The mean absolute difference was 0.62 in the face-down group and 0.34 in the no posture patients. Only two eyes $(6.7 \%)$ had an error of more than 1D from their intended refraction.

\section{Discussion}

Although this is a small trial, it provides valuable information for the design of larger more definitive studies. It has been suggested that combined phacoemulsification and vitrectomy allows for a larger gas bubble, as the volume of the intraocular lens is less than the volume of the normal lens. Cataract surgery causes a prolonged breakdown of the blood-aqueous barrier as measured by a laser flare metre..$^{22}$ Upregulation of cytokines caused by the inflammation might promote macular hole closure. In a large randomised trial of posturing for macular hole, patients who had combined surgery did not have a higher success rate than those who had vitrectomy alone. ${ }^{16}$

One argument against combined surgery is that biometry may be less accurate in the presence of macular disease that reduces central vision. ${ }^{23}$ In common with some other authors we found a slight myopic overcorrection, which might be explained by an anterior displacement of the capsular bag caused by the gas bubble..$^{24}$ Overall, the refractive outcomes were acceptable, with only two eyes (6.7\%) being more than 1D different from the intended spherical error. With modern biometric techniques it seems possible to achieve accurate lens power predictions, even in the presence of a macular hole. ${ }^{25}$

Visual outcomes were good, with $80 \%$ of eyes achieving $6 / 18$ or better vision, and every eye in which the hole closed able to read N10 or better. 
A possible weakness of this study is that we did not monitor patient posturing after surgery. Some patients in the no posture group may have decided to posture face down on their own initiative. It is likely that some of the patients randomised to face-down posture were unable to maintain the face-down position for 10 days. However this reflects normal clinical practice, in which surgeons are only able to advise their patients regarding postoperative positioning.

In this trial, the success rate of combined surgery without posturing was higher than in our companion study of vitrectomy alone, although with face-down posturing, the success rates were identical. While we attempted to ensure that similar surgical techniques were used for both studies, we cannot be sure that the study populations were identical.

Although the success rate was higher in the nonposturing arm of this pilot study, the confidence intervals around the estimates of success in the two arms do not exclude an increased risk of failure of up to $16.6 \%$ in the non-posturing arm. Using Pocock's formula, ${ }^{26}$ we estimate that 798 patients would be required to be $90 \%$ sure that the $95 \%$ confidence interval around the estimated difference in success rates when posturing and non-posturing will exclude a difference of more than 5\%. As the effect of posturing appears to be very different in combined surgery compared with vitrectomy alone, we recommend that future trials of posturing in macular hole surgery should randomise for combined vs sequential cataract surgery, as well as postoperative posturing.

\section{Summary}

What was known before

- Macular hole surgery has a high success rate with face-down posturing, but posturing is difficult for the patient and may have adverse effects.

- The importance of posturing for anatomical success is uncertain, although there is some evidence that it is not necessary in holes of $<400 \mu \mathrm{m}$ diameter. Recent systematic reviews have called for more randomised clinical trials.

What this study adds

- $29 / 30$ holes $(96.7 \%)$ were closed with a single operation, and $80 \%$ of eyes achieved $6 / 18$ or better.

- We were unable to show any difference between the posture and non-posture arms, but, because of the small numbers included, we cannot exclude the possibility that posturing may have a beneficial effect. A conclusive trial would require 798 patients.

\section{Conflict of interest}

The authors declare no conflict of interest.

\section{References}

1 Tranos PG, Ghazi-Nouri SM, Rubin GS, Adams ZC, Charteris DG. Visual function and subjective perception of visual ability after macular hole surgery. Am J Ophthalmol 2004; 138: 995-1002.

2 Hirneiss C, Neubauer AS, Gass CA, Reiniger IW, Priglinger SG, Kampik A et al. Visual quality of life after macular hole surgery: outcome and predictive factors. Br J Ophthalmol 2007; 91: 481-484.

3 Kelly NE, Wendel RT. Vitreous surgery for idiopathic macular holes. Results of a pilot study. Arch Ophthalmol 1991; 109: 654-659.

4 Madgula IM, Costen M. Functional outcome and patient preferences following combined phaco-vitrectomy for macular hole without prone posturing. Eye (Lond) 2008; 22: 1050-1053.

5 Dhawahir-Scala FE, Maino A, Saha K, Mokashi AA, McLauchlan R, Charles S. To posture or not to posture after macular hole surgery. Retina 2008; 28: 60-65.

6 Salam A, Harrington P, Raj A, Babar A. Bilateral Ulnar nerve palsies: an unusual complication of posturing after macular hole surgery. Eye 2004; 18: 95-97.

7 Sutter FK, Smorgon A, McClellan K. Acute angle closure in the fellow eye as a complication of prone positioning after vitreoretinal surgery. Arch Ophthalmol 2003; 121: 1057.

8 Gupta D. Face-down posturing after macular hole surgery: a review. Retina 2009; 29: 430-443.

9 Tornambe PE, Poliner LS, Grote K. Macular hole surgery without face-down positioning. A pilot study. Retina 1997; 17: 179-185.

10 Ellis JD, Malik TY, Taubert MA, Barr A, Baines PS. Surgery for full-thickness macular holes with short-duration prone posturing: results of a pilot study. Eye (Lond) 2000; 14: 307-312.

11 Mittra RA, Kim JE, Han DP, Pollack JS. Sustained postoperative face-down positioning is unnecessary for successful macular hole surgery. $\mathrm{Br} J$ Ophthalmol 2009; 93: 664-666.

12 Rubinstein A, Ang A, Patel CK. Vitrectomy without postoperative posturing for idiopathic macular holes. Clin Experiment Ophthalmol 2007; 35: 458-461.

13 Simcock PR, Scalia S. Phacovitrectomy without prone posture for full thickness macular holes. $\mathrm{Br} J$ Ophthalmol 2001; 85: 1316-1319.

14 Heath G, Rahman R. Combined 23-gauge, sutureless transconjunctival vitrectomy with phacoemulsification without face down posturing for the repair of idiopathic macular holes. Eye (Lond) 2010; 24: 214-220, quiz.

15 Tranos PG, Peter NM, Nath R, Singh M, Dimitrakos S, Charteris $\mathrm{D}$ et al. Macular hole surgery without prone positioning. Eye 2007; 21: 802-806.

16 Guillaubey A, Malvitte L, Lafontaine PO, Jay N, Hubert I, Bron A et al. Comparison of face-down and seated position after idiopathic macular hole surgery: a randomized clinical trial. Am J Ophthalmol 2008; 146: 128-134.

17 Tadayoni R, Vicaut E, Devin F, Creuzot-Garcher C, Berrod JP, Le Mer Y et al. A randomized controlled trial of alleviated positioning after small macular hole surgery. Ophthalmology 2011; 118: 150-155.

18 Tatham A, Banerjee S. Face-down posturing after macular hole surgery: a meta-analysis. Br J Ophthalmol 2010; 94: 626-631. 
19 Christensen UC, Krøyer K, Sander B, Larsen M, Henning V, Villumsen $\mathrm{J}$ et al. Value of internal limiting membrane peeling in surgery for idiopathic macular hole stage 2 and 3: a randomised clinical trial. Br J Ophthalmol 2009; 93: 1005-1015.

20 Jaycock PD, Bunce C, Xing W, Thomas D, Poon W, Gazzard $\mathrm{G}$ et al. Outcomes of macular hole surgery: implications for surgical management and clinical governance. Eye 2005; 19: 879-884.

21 Lange CAK, Membrey L, Ahmad N, Wickham L, Maclaren RE, Solebo L et al. Pilot randomised controlled trial of facedown positioning following macular hole surgery. Eye 2012; 26: 272-277.
22 Siriwardena D, Kotecha A, Minassian D, Dart JK, Khaw PT. Anterior chamber flare after trabeculectomy and after phacoemulsification. Br J Ophthalmol 2000; 84: 1056-1057.

23 Daniel C, Tuft S, Ionides A, Bunce C. Effect of visual acuity on biometry prediction error after cataract surgery. J Cataract Refract Surg 2003; 29: 1365-1369.

24 Patel D, Rahman R, Kumarasamy M. Accuracy of intraocular lens power estimation in eyes having phacovitrectomy for macular holes. J Cataract Refract Surg 2007; 33: 1760-1762.

25 Manvikar SR, Allen D, Steel DH. Optical biometry in combined phacovitrectomy. J Cataract Refract Surg 2009; 35: 64-69.

26 Pocock SJ. Clinical Trials: A Practical Approach. Wiley-Blackwell: Edinburgh, 1983. 